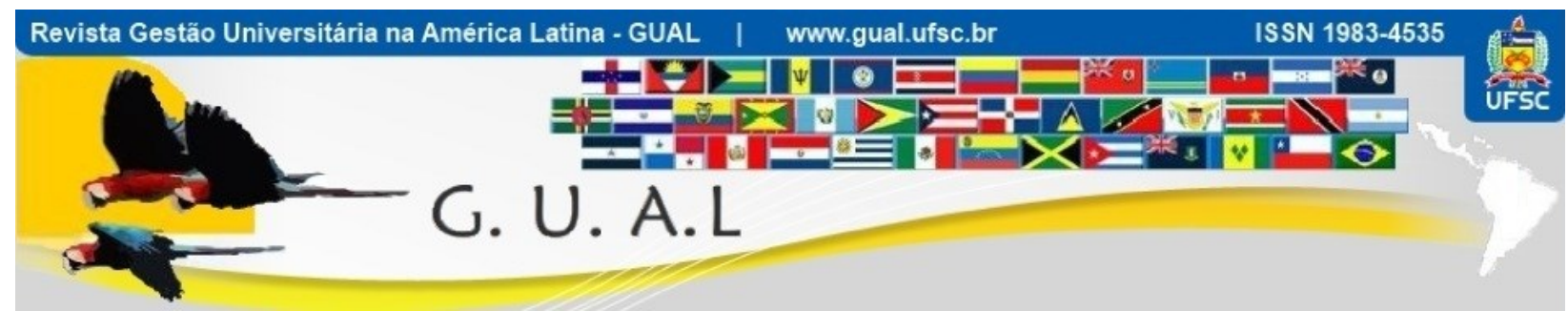

DOI: http://dx.doi.org/10.5007/1983-4535.2019v12n2p118

\title{
AVALIAÇÃO DA ACEITAÇÃO DAS FERRAMENTAS TECNOLÓGICAS NO AMBIENTE DO TRABALHO DOCENTE
}

\section{ACCEPTANCE OF ASSESSMENT OF TECHNOLOGICAL TOOLS IN TEACHER ACADEMIC WORK ENVIRONMENT}

\author{
Alberto Luís da Silva Pinto, Especialista \\ https://orcid.org/0000-0002-5996-0204 \\ alberto.livemocha@gmail.com \\ Universidade Federal do Piauí | Centro Ciências Agrárias \\ Teresina | Piauí | Brasil
}

Elvia Florencio Torres, Mestre https://orcid.org/0000-0001-7750-8468 elviaftorres@gmail.com Universidade Federal do Piauí | Centro de Educacao Aberta e a Distância Teresina | Piauí | Brasil

Joária Mendes de Moura, Graduada https://orcid.org/0000-0002-6003-0311 joariamoura@gmail.com Universidade Federal do Piauí | Centro de Educacao Aberta e a Distância Teresina | Piauí | Brasil

Evangelina da Silva Sousa, Mestre https://orcid.org/0000-0002-8455-1824 evangelinasousa@gmail.com

Universidade Federal do Piauí | Centro de Educacao Aberta e a Distância Teresina | Piauí | Brasil

Liliane Araújo Pinto, Mestre https://orcid.org/0000-0003-3804-2540

liliane@ufpi.edu.br Universidade Federal do Piauí | Centro de Ciências da Educação Teresina | Piauí | Brasil

Recebido em 05/fevereiro/2018

Cléverson Vasconcelos da Nóbrega, Doutor https://orcid.org/0000-0001-8173-2868 cleverson.nobrega@gmail.com Aprovado em 20/julho/2018 Universidade Federal do Piauí | Campus Senador Helvídio Nunes de Barros Publicado em 02/maio/2019

Picos | Piauí | Brasil

Sistema de Avaliação: Double Blind Review

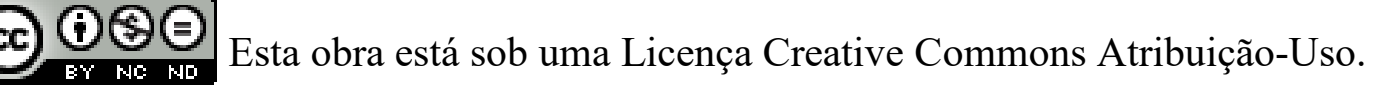




\title{
RESUMO
}

A pesquisa objetivou avaliar a interface do trabalhador com as tecnologias aplicadas no ambiente da Educação a Distância $(\mathrm{EaD})$, analisando o grau de aceitação e utilização efetiva do Sistema Integrado de Gestão de Atividades Acadêmicas (SIGAA) do Centro de Educação Aberta e à Distância (CEAD) da Universidade Federal do Piauí (UFPI) por meio dos construtos do Modelo de Aceitação de Tecnologia (TAM) de Davis (1989). A pesquisa foi aplicada a 83 professores tutores, distribuídos em 16 cursos. Para o tratamento dos dados foram utilizadas técnicas de análise multivariadas e análise descritiva quantitativa e qualitativa. Além disso, a pesquisa apresentou um alto índice de confiabilidade. Constatou-se que os professores-tutores que atuam na modalidade à distância possuem uma boa aceitação ao SIGAA por perceberem a Utilidade e a Facilidade de Uso do Sistema, o que corrobora efetivamente pela Atitude e Intenção Comportamental para o Uso Real da Tecnologia.

Palavras-chave: Modelo de Aceitação de Tecnologia. Sistema de Informação. Educação a Distância.

\begin{abstract}
This research aimed to assess the worker interface with applied technologies into Distance Education environment (EaD), as well as measuring level of acceptance and effective use of the Integrated System of Management of Academic Activities (SIGAA) from the Open and Distance Education Center (CEAD) at Federal University of Piaui (UFPI). It was performed by constructs of the Technology Acceptance Model (TAM) by Davis (1989). The survey was applied to 83 tutors-professors distributed in 16 courses. It was used multivariate analysis techniques and quantitative-qualitative descriptive analysis for treatment of the data. Furthermore this paper presented a high reliability level (Alph de Cronbach de 0,83). Therefore, the tutors from the distance modality claim that are satisfied with the SIGAA, because they noted the use and easily of the System. It effectively is corroborated by Attitude and Behavioral Intent for actual use of the technology.
\end{abstract}

Keywords: Technology Acceptance Model, Information System, Distance Education. 


\section{INTRODUÇÃO}

$\mathrm{Na}$ Era da informação, as mudanças de modelos e padrões, os novos paradigmas, em todos os tipos de ambientes, promovidas pela Globalização e Revolução Digital, desde o limiar do século XXI, continuam evoluindo e provocando a sociedade mudanças de muitas concepções tradicionais em todos os campos. Nesse cenário, a Tecnologia da Informação (TI) tem sido uma realidade cada vez mais frequente nas organizações, desafiando os profissionais a uma nova cultura.

Os benefícios da TI no ambiente organizacional são a possibilidade de implantar novas estratégias, especialmente, aquelas que focam a exploração de novos modelos, ferramentas e oportunidades na inovação de produtos e serviços. As Tecnologias de Informação e Comunicação (TICs) estão presentes em todos os processos administrativos e produtivos. De acordo com Limongi-França (2009, p. 51): "atualmente, a tecnologia de informação e comunicação está aumentando o número de empregos em todo o mundo, além de elevar as taxas de crescimento e produtividade e de reduzir os custos das empresas".

No ambiente da Educação a Distância $(\mathrm{EaD})$, o advento das TICs trouxe novas perspectivas devido às facilidades de design e produtos sofisticados, rápida emissão e distribuição de conteúdos, interação com informações, recursos e pessoas, além da flexibilidade do tempo e da quebra de barreiras espaciais, criando um ambiente estratégico para democratizar e elevar o padrão de qualidade da formação de profissionais e a melhoria da educação brasileira (ALMEIDA, 2012).

Nesse contexto, a aceitação da tecnologia no ambiente de trabalho docente é um tema que merece a atenção de estudiosos e profissionais das mais diversas áreas do conhecimento. Conforme Silva et al. (2013): vários modelos teóricos têm sido desenvolvidos e aplicados para estudar a aceitação e o comportamento de uso de TI, entretanto, entre as diversas teorias propostas, o Modelo de Aceitação de Tecnologia, Technology Acceptance Model (TAM), é considerado um dos mais influentes e mais amplamente utilizado pelos pesquisadores para descrever a aceitação, de determinada tecnologia, pelos indivíduos, estudando a influência de fatores humanos na adoção de novas ferramentas tecnológicas.

O objetivo da pesquisa foi avaliar a interface do trabalhador com as tecnologias digitais aplicadas no ambiente da $\mathrm{EaD}$ com foco no trabalho docente de professores-tutores a distância, analisando o grau de aceitação e utilização efetiva do Sistema Integrado de Gestão de Atividades Acadêmicas (SIGAA) por meio dos construtos "Utilidade Percebida" 
(Perceived Usefulness) e "Facilidade de Uso Percebida" (Perceived Ease of Use) do TAM (DAVIS, 1989).

A relevância da pesquisa reside no fato de que ainda são incipientes os estudos realizados aplicando-se o TAM no contexto desses profissionais, o que torna ainda mais significativo e efetivo estudar e analisar a percepção dos professores-tutores a distância na utilização dos sistemas de informação (SI), considerando-se o modelo proposto por Davis (1989) por focar nos porquês dos usuários aceitarem ou rejeitarem a TI e como melhorar a aceitação, oferecendo desse modo, um suporte para prever e explicar a aceitação (SILVA et al., 2013).

\section{REFERENCIAL TEÓRICO}

\subsection{INSERÇÃO E ACEITAÇÃO DA TECNOLOGIA NO AMBIENTE DE TRABALHO}

O mundo está vivenciando, através da TI, uma mudança tecnológica como foram aquelas que precederam as grandes revoluções industriais pela "sua penetração em todos os domínios da atividade humana, não como força exógena de impacto, mas como tecido em que essa atividade é exercida" (CASTELLS, 2000, p. 50).

Os impactos positivos ou negativos da inserção das tecnologias nas organizações podem ser semelhantes ou distintos nos ambientes organizacionais, logo os impactos ocorridos no ambiente do trabalho docente nas instituições de ensino precisam ser analisados, pois as TICs são poderosas ferramentas para o desenho de processos de negócios e a força estratégica está na capacidade de ampliar relacionamentos em redes e formas virtuais de organização. Assim, difunde-se cada vez mais a $\mathrm{EaD}$, as bibliotecas virtuais, o correio eletrônico, a videoconferência, os portais corporativos e os aplicativos de apoio às atividades acadêmicas desempenhadas pelos docentes nas Instituições de Ensino Superior (IES), o que impele dedicação e estudo.

Sabe-se que a função principal de toda e qualquer tecnologia é aperfeiçoar as condições de vida ou de trabalho de um ou de vários indivíduos, mediante utilização de instrumentos, mecanismos ou procedimentos que promovam a ação humana. Nesse sentido, importa ressaltar que muitos estudos têm sido realizados com o propósito de entender a relação existente entre indivíduos e processos tecnológicos (CARVALHO, 2013).

Segundo Teo (2011, p. 1): o construto 'aceitação da tecnologia' pode ser entendido como "a boa vontade do usuário em empregar a tecnologia nas tarefas para as quais ela foi 
projetada para dar suporte". Assim, os processos de construção de novas tecnologias educacionais precisam considerar os estudos relacionados à aceitação de tecnologias para que possam agregar soluções facilitadoras e essas contribuam para a aceitação e uso dessas tecnologias (SILVA, 2014).

Observa-se que na literatura da ciência da computação são comuns os estudos voltados para os componentes técnicos dos sistemas, por outro lado a ciência da informação veio modificar essa abordagem, dando mais importância ao uso efetivo dos sistemas e dos atributos de qualidades percebidas pelos usuários. Entender porque as pessoas usam ou rejeitam computadores tornou-se um dos mais desafiadores temas em pesquisas sobre os sistemas de informação (SILVA et al., 2013).

Silva et al. (2012) destacam a importância de não se ter apenas um olhar técnico, isto é, direcionar as atenções aos requisitos oferecidos pela tecnologia, para entender a utilização da TI, mas sim buscar compreender o comportamento dos usuários. Ressaltam Venkatesh et al. (2003) que a aceitação da tecnologia vem sendo estudada há mais de duas décadas, ocasionando a proliferação de inúmeros modelos que buscam esclarecer a adoção da tecnologia individual. Os autores destacam que entender e criar as condições sob as quais os SI são adotados pelas organizações permanece sendo uma área de pesquisa de alta prioridade.

Vários são os estudos e modelos desenvolvidos e aplicados para analisar a aceitação dos usuários na adoção de novas tecnologias nas organizações, entre eles, destacam-se a Teoria da Ação Intencional (TRA) de Fishbein e Ajzen (1975); o Modelo de Aceitação Tecnológica (TAM) de Davis (1989); a Extensão do Modelo de Aceitação Tecnológica (TAM2) e o Modelo Integrado de Aceitação Tecnológica (TAM3), ambos de Davis et al. (1989); a Teoria Unificada de Aceitação e Utilização de Tecnologia (UTAUT) de Venkatesh et al. (2003), conforme Bradley (2009) e Carvalho et al. (2013).

Desse modo, é possível identificar diversas teorias que tentam prever o impacto da tecnologia no comportamento humano, entretanto, a pesquisa focou-se no TAM original, modelo plenamente aplicável ao objetivo do estudo, por ser específico para os usuários de SI e por apresentar a vantagem de possuir uma forte base teórica e um amplo apoio empírico através de validações, aplicações e replicações, conforme vasta literatura sobre o modelo.

Nesse particular, a EAD evoluiu com grande velocidade com as novas tecnologias, consolidando o ambiente de trabalho docente dos professores-tutores. No princípio, estava limitada principalmente pelo tipo de conexão do usuário, assim como de seu acesso aos meios 
tecnológicos. As últimas décadas foram ainda mais importantes para o crescimento da $\mathrm{EaD} \mathrm{e}$ os avanços experimentados pelas TICs foram fundamentais. Assim, a revolução digital transforma o espaço educacional, pois na Era digital, o saber é potencializado pelas estradas virtuais da informação (TEZZA, 2012).

\subsection{TECHNOLOGY ACCEPTANCE MODEL (TAM)}

O Technology Acceptance Model (TAM) é uma adequação da teoria da atuação racional, derivada da psicologia e alterada especificamente para gerar modelos de aceitação de tecnologia da informação. A teoria proveniente da psicologia é a Teoria da Ação Racionalizada (TRA), que diz que o comportamento de determinado indivíduo em uma situação particular é influenciado pela intenção comportamental de uso em evidenciar ou exercer tal comporTAMento, que por sua vez é distinguida por sua atitude e normas subjetivas (CARVALHO, 2013).

Nesse contexto, o modelo tem por objetivo auxiliar os responsáveis pela implantação de SI e avaliar sua aceitação presente e futura, isto é, pretende explicar o motivo de alguns utilizadores aceitarem e outros rejeitarem os SI introduzidos no seu local de trabalho (CARVALHO, 2013).

Para Davis (1989) o TAM possibilita um esclarecimento acerca dos determinantes da utilização de computadores, capaz de considerar comportamentos de usuários através de um grande arsenal de tecnologias e populações. O modelo sustenta a ideia de que os estímulos externos influenciam as atitudes pessoais, influenciando indiretamente suas crenças sobre as consequências de ter aquele comportamento. Assim, um ponto central do TAM é examinar o impacto de fatores externos nas crenças e atitudes das pessoas.

O modelo TAM foi projetado para compreender a relação causal entre variáveis externas de aceitação dos usuários e o uso real do computador, buscando compreender o comportamento destes usuários através do conhecimento da utilidade e da facilidade de utilização percebida por eles (DAVIS, 1989).

A teoria do TAM baseia-se em dois conceitos principais: a utilidade percebida e a facilidade de uso percebida. Esses dois construtos são capazes de prever a aceitação de sistemas computacionais. Conforme Davis (1989), os indivíduos tendem a utilizar ou não determinada aplicação ou tecnologia de acordo com a possibilidade de melhorar seu desempenho no trabalho. Em suma, o modelo apresenta uma base para delinear o impacto das 
variáveis em crenças internas, atitudes e intenções (DAVIS, 1989). A Figura 1 mostra o modelo original do TAM, conforme Park (2009).

Figura 1 Original do Modelo de Aceitação de Tecnologia (TAM).

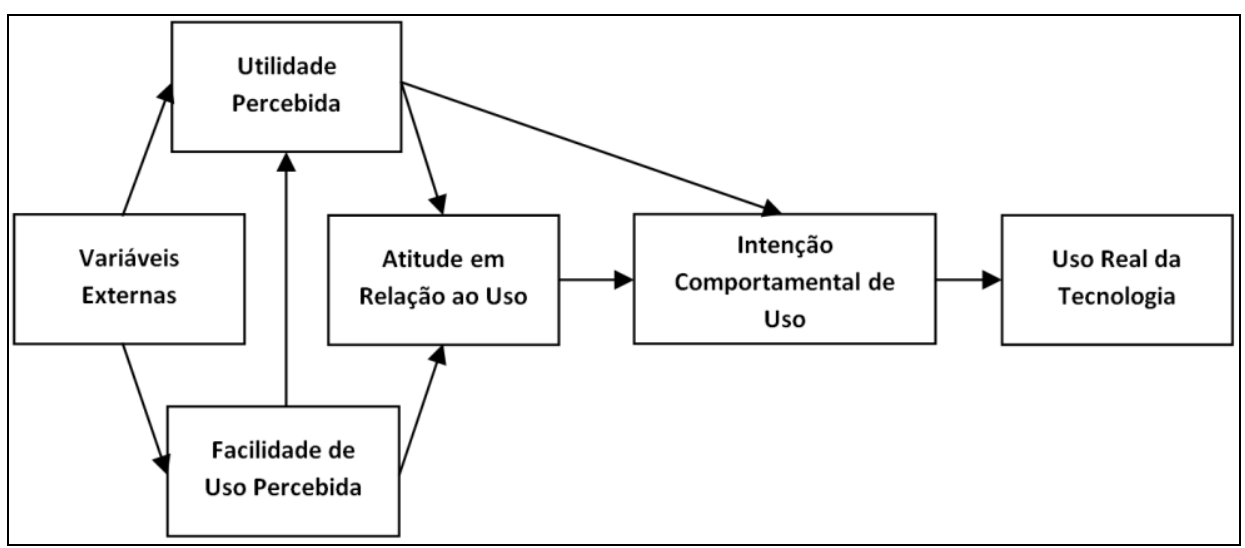

Fonte: Park, 2009.

Como posto anteriormente, Davis (1989) define os dois principais determinantes do TAM da seguinte forma: a Utilidade Percebida (Perceived Usefulness) é o grau em que o usuário acredita que o uso de um sistema particular pode melhorar o seu desempenho profissional; e a Facilidade de Uso Percebida (Perceived Ease of Use) é o grau em que o usuário acredita que o uso de um sistema de informação será livre de dificuldade, ou seja, será fácil de ser manuseado.

Cabe aqui se explicitar ainda o construto Atitude que, segundo Fishbein \& Ajzen (1975, p. 216 apud CARVALHO, 2013), é caracterizado como "sentimentos positivos ou negativos de um individuo sobre a realização de um determinado comportamento". Os autores definem, também, o construto Intenção Comportamental que é estabelecido a partir da intensidade de o usuário realizar um comportamento específico.

Segundo Bueno et al. (2004): o construto Variáveis Externas refere-se às características do sistema, processo de desenvolvimento, treinamento etc. Enquanto que o construto Uso Real do Sistema é a quantidade de uso sobre uma unidade fixa de tempo, que pode ser medido pela próprio sistema internamente ou pela manifestação do usuário.

Desse modo, a Utilidade Percebida sofre influência direta da Facilidade Percebida e ambas influenciam a Atitude que um indivíduo terá ao utilizar determinado sistema. A Atitude é a maneira como o individuo se comporta, procede ou age. Dentro do contexto do TAM, a Atitude representa o desejo do usuário de utilizar o sistema. A Utilidade Percebida e a Atitude em Relação ao Uso influenciam a Intenção Comportamental de Uso - intenção de 
usar o sistema no futuro. A Intenção determinará o Uso Real do Sistema (CARVALHO, 2013).

Sendo o TAM um modelo comportamental, deve referir-se aos usuários e suas percepções sobre o uso de determinado sistema, desse modo, os construtos devem ser desenvolvidos para captar opiniões pessoais e tratar suposições a respeito das pessoas ou instituições. O modelo é útil para identificar o porquê da não aceitação de um sistema ou tecnologia em particular pelos usuários e, consequentemente, implementar os passos corretivos adequados para solucionar essa situação (SILVA et al., 2012).

\subsection{Sistema Integrado de Gestão de Atividades Acadêmicas (SIGAA)}

Percebe-se que a implementação de SI nas organizações, como é o caso dos Sistemas Integrados de Gestão (SIG), geralmente produz mudanças significativas, pois alteram a forma como as pessoas estão habituadas a realizar suas atividades, exigindo uma perspectiva cada vez mais multifacetada e complexa dos processos de trabalho, uma nova relação de poder e hierarquia, além de novo nível e tipo de conhecimento tecnológico. Para a efetivação dessas mudanças tecnológicas, alguns aspectos são essenciais, entre eles, a ação dos profissionais como colaboradores ativos de uma gestão flexível e um SIG que propicie essa nova configuração de organização do trabalho, possibilitando o controle corporativo do negócio (TENÓRIO, 2007).

Segundo Machado et al. (2011): as tecnologias de comunicação foram incorporadas à TI como resultado de melhoria e inovação, surgindo assim as TICs. A inserção das tecnologias no ambiente do trabalho docente proporcionou como consequência a propagação das TICs influenciando o ambiente de ensino, levando a novas necessidades educacionais e possibilidades metodológicas e criando modelos criativos de aprendizagem, onde a ação do docente e do discente é parcialmente diferente dos padrões considerados tradicionais (HUERTAS, 2007).

Nesse ambiente, a Superintendência de Informática (SINFO) da Universidade Federal do Rio Grande do Norte (UFRN), desenvolveu o SIGAA com o objetivo de informatizar os procedimentos da área acadêmica, envolvendo ensino, pesquisa, extensão e gestão. O SIGAA também disponibiliza portais específicos para professores, alunos e tutores do ensino a distância, entre os quais a Turma Virtual, que é uma ferramenta de ensino complementar colocada à disposição dos docentes e discentes, como um espaço construído para ajudar no 
aprendizado dos discentes, criando uma extensão da sala de aula e permitindo o intercâmbio virtual de informações entre professores e alunos de uma turma (UFRN, 2016).

Ainda assim, Chen (2004) entende que os docentes não se sentem encorajados em utilizar computadores no formato em que vêm sendo demandados, o que é um desafio à ideia de que a tecnologia promova melhorias no processo ensino-aprendizagem. Conforme o autor, para que os investimentos em tecnologia na educação possam gerar retornos é necessário que os docentes apresentem atitude positiva em torno dos computadores e se sintam motivados a integrar a tecnologia em suas estratégias pedagógicas.

\section{METODOLOGIA}

Esta pesquisa classifica-se como quantitativo-qualitativa quanto ao método e à forma de abordar a problemática. Primeiramente, utilizou-se de instrumentos estatísticos, tanto na coleta como no tratamento dos dados com a finalidade de mensurar as relações entre as variáveis, o que caracteriza a pesquisa quantitativa por ser apropriada para medir tanto opiniões, atitudes e preferências como comportamentos. Depois, preocupou-se em descrever os fenômenos por meio dos significados que o próprio ambiente natural manifesta, caracterizando a pesquisa como qualitativa por analisar informações teórico-empíricas que permitem atribuir-lhe cientificidade (ZANELLA, 2009).

Quanto aos objetivos, a pesquisa é descritiva e exploratória. Primeiro, porque é apropriada para descrever as características de determinado fato ou fenômeno. Segundo, porque é centrada na preocupação de identificar fatores determinantes ou contributivos ao desencadeamento dos fenômenos (ZANELLA, 2009).

Utilizou-se, quanto aos procedimentos adotados na coleta de dados, a pesquisa por levantamento (surveys, ou sondagem), pois se coletaram os dados junto ao público objeto de estudo através da ferramenta questionários, construídos com base nos indicadores de avaliação existentes na literatura. Segundo Oliveira (2011): o questionário é uma “[...] série ordenada de perguntas, respondidas por escrito sem a presença do pesquisador".

Para elaboração do questionário, utilizou-se uma escala Likert com 5 (cinco) níveis de concordância, variando desde o "discordo totalmente" até o "concordo totalmente", para se analisar as percepções dos respondentes quanto às ferramentas disponibilizadas pela plataforma on-line da EaD - o SIGAA. Os questionamentos tiveram como base o Modelo TAM de Davis (1989). 
O questionário foi enviado através de formulário eletrônico do Google aos professores-tutores que atuam na modalidade a distância junto ao Centro de Educação Aberta e à Distância (CEAD) da Universidade Federal do Piauí (UFPI), distribuídos nos pólos dos Estados da Bahia, Pernambuco e Piauí, totalizando 306 (trezentos e seis) profissionais vinculados aos 16 (dezesseis) cursos de graduação a distância, quais sejam: administração, biologia, ciências da natureza, computação, ecologia, filosofia, física, geografia, gestão pública, história, inglês, matemática, pedagogia, português, química e sistema de informação.

Após a coleta e análise dos dados junto ao público objeto da pesquisa, realizou-se presencialmente uma entrevista semiestruturada com os coordenadores dos cinco cursos com maior número de professores-tutores, ou seja, administração, filosofia, letras-português, letras-inglês e sistema de informação, com o objetivo de compreender a percepção desses profissionais da gestão quanto à familiaridade e às dificuldades dos professores-tutores na utilização do SIGAA, confrontando o posicionamento destes com os resultados obtidos através do questionário aplicado com os professores-tutores.

No tratamento estatístico, considerou-se um erro amostral de $10 \%\left(\mathrm{E}_{0}=10 \%\right)$. Para calcular a primeira aproximação da amostra utilizou-se a fórmula: $\mathrm{n}_{0}=1 / \mathrm{E}^{2}$, chegando-se ao número de 100 pessoas. Para o cálculo total da amostra os dados da população foram lançados na fórmula: $\mathrm{n}=\mathrm{N} \cdot \mathrm{n}_{0} / \mathrm{N}+\mathrm{n}_{0}$, sendo $\mathrm{N}$ a população (306 professores-tutores da modalidade a distância) e $n_{0}$ o valor calculado anteriormente. Assim, o resultado final foi uma amostra desejada de 75 participantes. A amostra real foi de 83 profissionais distribuídos aleatoriamente entre os cursos do CEAD, conforme Tabela 1, perfazendo um número estatisticamente aceitável.

O período de coleta junto à população ocorreu no intervalo de 17 (dezessete) dias corridos, sendo de 23/05 a 07/06/2016, tendo se atingido uma participação de 27,12\% (vinte e sete vírgula doze por cento) do total de professores-tutores a distância do CEAD/UFPI e as entrevistas com os coordenadores dos cursos aconteceram presencialmente no dia 22/06/2016 no CEAD/UFPI.

A tabulação dos dados foi feita por meio do Software SPSS, versão 20 que permitiu validar e adaptar o Modelo TAM à realidade estudada. Duas técnincas de análise multivariada foram aplicadas, Análise Fatoria Exploratória (AFE), e a regressão multinominal. A Análise Fatoria Exploratória (AFE), que consiste em uma técnicas de análise multivariada que foi aplicada com o objetivo de reduzir as variáveis do modelo à algumas categorias, bem como 
verificar o comportamento dessas variáveis em paralelo com a teoria sugerida por Davis (1989), podendo dessa forma, adaptar o modelo a realidade estudada.

Tabela 1 Quantitativo dos tutores da modalidade a distância do CEAD/UFPI.

\begin{tabular}{clccc}
\hline Ord & \multicolumn{1}{c}{ Cursos } & $\begin{array}{c}\text { Quantidade } \\
\text { Tutores }\end{array}$ & $\begin{array}{c}\text { Quantidade } \\
\text { Respostas }\end{array}$ & Percentual (\%) \\
\hline 1. & Filosofia & 45 & 18 & 40,00 \\
2. & Administração & 33 & 10 & 30,30 \\
3. & Gestão Pública & 12 & 04 & 33,33 \\
4. & Química & 20 & 03 & 15,00 \\
5. & Matemática & 17 & 04 & 23,53 \\
6. & Sistema de Informação & 38 & 08 & 21,05 \\
7. & Letras - Português & 28 & 11 & 39,29 \\
8. & Ecologia & 01 & 00 & 00,00 \\
9. & Ciências da Natureza & 02 & 01 & 50,00 \\
10. & Biologia & 01 & 01 & 100,00 \\
11. & Computação & 14 & 04 & 28,57 \\
12. & Geografia & 23 & 07 & 30,43 \\
13. & Letras - Inglês & 27 & 02 & 7,41 \\
14. & História & 09 & 01 & 11,11 \\
15. & Pedagogia & 18 & 04 & 22,22 \\
16. & Física & 18 & 05 & 27,78 \\
\hline & TOTAIS & $\mathbf{3 0 6}$ & $\mathbf{8 3}$ & $\mathbf{2 7 , 1 2}$ \\
\hline
\end{tabular}

Fonte: dados da pesquisa, jun. 2016.

Após o processamento da AFE, observou-se que as primeiras variáveis se agruparam conforme sugerido pelo modelo original nas categorias: Facilidade de uso percebida e Utilidade percebida. Porém as duas categorias seguintes (Atitude no sentido de usar e Intensão comportamental para usar) se fundiram formando o constructo que se denominou de: Importância direcionada a ação. A ultima categoria apresentou uma variável sem significância além disso, houve uma que migrou da categoria: Intenção comportamental para usar, formando o constructo: Disposição comportamental para uso real do sistema. O Quadro 1, apresenta esses dados de forma mais sistemática.

Por fim, foi calculado o Alfa de Cronbach, que corresponde à medida de confiança da pesquisa. Esse indicador, pode variar de 0 a 1 . Quanto mais próximo a 1, maior será a confiabilidade, sendo os valores de 0,6 a 0,7 considerados o limite de aceitabilidade (HAIR JR. et al 2005). O Alph de Cronbach da pesquisa foi de 0,83, o que torna os dados confiáveis.

A análise dos dados foi de modo quantitativo e qualitativo, para tanto aplicaram-se análises estatíticas para a formulação de gráficos e tabelas, envolvendo as quatro categorias avaliadas listadas no Quadro 01. 
Quadro 1 Categorias do modelo TAM anttes e depois da AFE.

\begin{tabular}{|c|c|c|}
\hline $\begin{array}{l}\text { Categorias do } \\
\text { modelo original }\end{array}$ & Variáveis & Novas Categorias \\
\hline \multirow{3}{*}{$\begin{array}{l}\text { Facilidade de uso } \\
\text { percebida }\end{array}$} & Acho o Sistema de Informação SIGAA fácil de usar & \multirow{3}{*}{ Facilidade de uso percebida } \\
\hline & Aprender a usar o SIGAA foi fácil para mim. & \\
\hline & É fácil tornar-se hábil em usar o SIGAA. & \\
\hline \multirow{3}{*}{ Utilidade percebida } & Utilizar o SIGAA melhora a qualidade do meu trabalho. & \multirow{3}{*}{ Utilidade percebida } \\
\hline & Utilizar o SIGAA aumenta minha produtividade no trabalho. & \\
\hline & $\begin{array}{l}\text { Utilizar o SIGAA é importante para a execução das minhas } \\
\text { atividades de professor-tutor. }\end{array}$ & \\
\hline \multirow{3}{*}{$\begin{array}{l}\text { Atitude no sentido de } \\
\text { usar }\end{array}$} & $\begin{array}{l}\text { Realizar minhas atividades através das ferramentas do } \\
\text { SIGAA é uma ideia inovadora. }\end{array}$ & \multirow{5}{*}{$\begin{array}{l}\text { Importância direcionada a } \\
\text { ação }\end{array}$} \\
\hline & $\begin{array}{l}\text { Considero o SIGAA e suas ferramentas um sistema de } \\
\text { informação positivo, eficiente e eficaz. }\end{array}$ & \\
\hline & $\begin{array}{l}\text { O SIGAA é compatível com outros sistemas de informação } \\
\text { que uso no meu cotidiano. }\end{array}$ & \\
\hline \multirow{3}{*}{$\begin{array}{l}\text { Intensão } \\
\text { comportamental para } \\
\text { usar }\end{array}$} & $\begin{array}{l}\text { Pretendo explorar ao máximo as ferramentas do SIGAA e } \\
\text { tornar-me um usuário expertise dessas funcionalidades. }\end{array}$ & \\
\hline & $\begin{array}{l}\text { Mesmo quando houver outras opções para a realização das } \\
\text { minhas atividades, o SIGAA será sempre minha primeira } \\
\text { opção no trabalho como professor-tutor. }\end{array}$ & \\
\hline & $\begin{array}{l}\text { Procuro resolver as pendências via SIGAA no dia em que } \\
\text { elas surgem. }\end{array}$ & \multirow{3}{*}{$\begin{array}{l}\text { Dosposição } \\
\text { comportamental para uso } \\
\text { real do sistema }\end{array}$} \\
\hline \multirow{3}{*}{ Uso real do sistema } & Atualizo as minhas atividades no SIGAA diariamente. & \\
\hline & $\begin{array}{l}\text { Cumpro a minha carga horária como professor-tutor todos os } \\
\text { dias online no SIGAA. }\end{array}$ & \\
\hline & $\begin{array}{l}\text { Executo alguma atividade suportada pelo SIGAA em outro } \\
\text { ambiente virtual. }\end{array}$ & Variável sem significância \\
\hline
\end{tabular}

Fonte: Elaborado pelos autores, maio de 2018.

\section{RESULTADOS E DISCUSSÕES}

\subsection{Perfil dos professores-tutores a distância do CEAD/UFPI}

A amostra indicou demograficamente predominância de professores-tutores do sexo masculino (61,4\%); faixa etária entre 25 e 40 anos (66,3\%); formação acadêmica de especialistas $(75,9 \%)$ e mestres $(15,7 \%)$; mais de cinco anos de experiência com o uso do computador (90,4\%); um a três anos de experiência com o uso do SIGAA $(68,7 \%)$; um a três anos de experiência na $\mathrm{EaD}(55,4 \%)$.

O treinamento para o uso do SIGAA foi realizado com $71,1 \%$ da amostra. No que diz respeito a esse aspecto, vale destacar que na fase de tratamento estatístico de dados, foi aplicada uma técnica de análise multivariada denominada regressão logística multinomina conforme já citado na parte metodológica deste artigo. Considerou-se como variável 
dependente a pergunta: "você recebeu treinamento? (sim ou não) e independentes os 4 fatores obtidos na AFE. Os resultados demonstraram que dois fatores possuem maior significância: a Facilidade de Uso Percebida, com efeito negativo, e a Importância Direcionada a Ação com efeito positivo. O que significa dizer que, mesmo sem treinamento o usuário percebe a facilidade no uso da ferramenta, porém o treinamento é importante para alinhar as ações para o uso do sistema por parte dos professores tutores.

Observou-se também que a maior concentração dos respondentes quanto à experiência com a $\mathrm{EaD}$, consequentemente, com o uso do SI, está entre os professores-tutores que se encontram no intervalo de um a três anos de atuação no ensino a distância do CEAD/UFPI, o que demonstra uma experiência ainda principiante no uso das ferramentas tecnológicas do sistema utilizado na instituição.

\subsection{Percepção dos professores-tutores quanto à Facilidade de Uso Percebida}

Para análise dessa categoria foram elaboradas as seguintes afirmações, nas quais os professores-tutores tiveram que manifestar concordância ou discordância: Acho o Sistema de Informação SIGAA fácil de usar (Q10); Aprender a usar o SIGAA foi fácil para mim (Q11); É fácil tornar-se hábil em usar o SIGAA (Q12). O posicionamento dos tutores está descrito no Gráfico 1.

Gráfico 1 Facilidade de Uso Percebida.

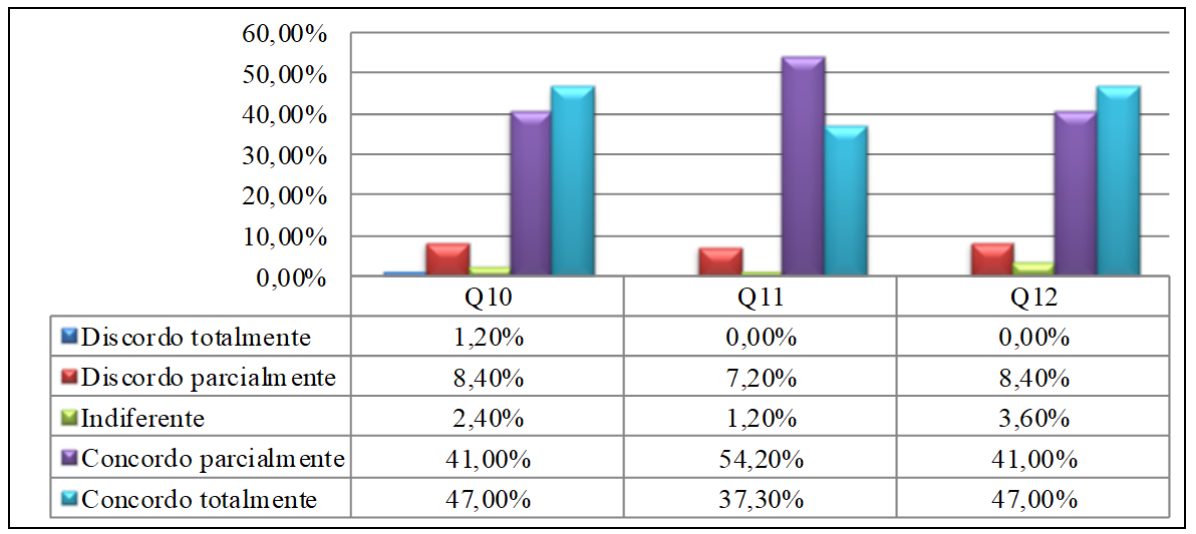

Fonte: dados da pesquisa, jun. 2016.

Com base no referido gráfico, pode-se inferir que os profissionais em questão consideram que o Sistema de Informação SIGAA apresenta o construto da Facilidade de Uso Percebida, pois, em média, $89,2 \%$ dos participantes afirmaram concordar parcial e totalmente com tais assertivas. 
Conforme preceitua a literatura, a percepção positiva dos profissionais quanto à Facilidade de Uso Percebida diante de um SI demonstra que variáveis externas como crenças, atitudes e intenções (DAVIS, 1989) possibilitam ao usuário vir a ter uma atitude e uma intenção comportamental de uso mais favorável ao uso efetivo da ferramenta tecnológica em seu ambiente de trabalho, pois se a ferramenta é de fácil manuseio o profissional estará mais predisposto em aceitá-la cotidianamente do que em rejeitá-la.

A interface dos sistemas é fator decisivo no processo de aprendizagem e domínio dos mesmos, sendo fator relevante no desenvolvimento e aprimoramento de ferramentas com recursos autoexplicativos. No tocante à facilidade de uso, o SIGAA apresenta uma interface onde as atividades são distribuídas em layouts de páginas que agrupam funcionalidades afins, distribuídas de forma clara, o que facilita a interação do usuário com o sistema. Essa facilidade é expressa no percentual acumulado das duas primeiras variáveis de Q10 (88\%), Q11 (91,5\%) e Q12 (88\%).

\subsection{Percepção dos professores-tutores quanto à Utilidade Percebida}

Os professores-tutores, que atuam na modalidade a distância, se manifestaram satisfeitos, quanto à Utilidade Percebida em relação ao SIGAA, ao se posicionarem de acordo com as seguintes proposições: Utilizar o SIGAA melhora a qualidade do meu trabalho (Q13); Utilizar o SIGAA aumenta a minha produtividade no trabalho (Q14); Utilizar o SIGAA é importante para a execução das minhas atividades de professor-tutor (Q15). O Gráfico 2 mostra que, em média, 95,2\% dos participantes afirmaram concordar parcial e totalmente com tais assertivas. Assim, percebe-se que o Sistema de Informação SIGAA apresenta de modo visível o construto da Utilidade Percebida.

Gráfico 2 Utilidade de Uso Percebida.

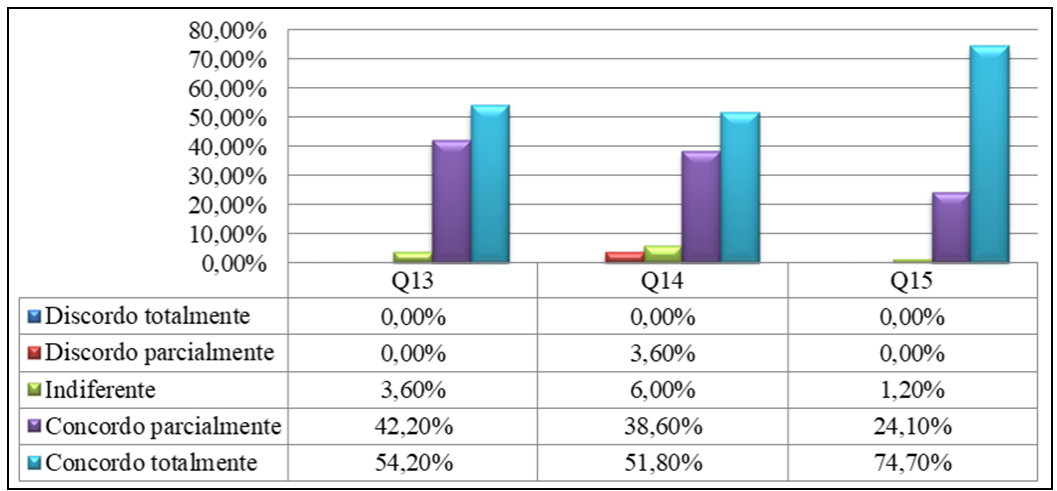

Fonte: dados da pesquisa, jun. 2016. 
A relevância em indagar aspectos relativos ao fator Utilidade reside nas funcionalidades que agregam valores ao SIGAA. Tais funcionalidades buscam facilitar a interação tutor/sistema e tutor/aluno através de ferramentas como chat, inserção de arquivos, vídeos, página da web, espaço para questionários e banco de questões, enquetes, estatísticas, correio para troca de mensagens entre tutores e alunos, twitter, manual de ajuda da turma virtual, Diário Eletrônico onde através do mesmo é possível ver o Diário de Turma, lançar a frequência, lançar notas, conteúdo programado da disciplina, a lista de presença, o mapa de frequência, o total de faltas por unidade etc.

Diante de um sistema de informação, se a percepção dos profissionais for positiva, em relação à Utilidade de Uso Percebida, isso demonstra a possibilidade do usuário vir a ter uma atitude e uma intenção comportamental de uso mais propícia a valorizar a ferramenta tecnológica em seu trabalho diário, pois se a ferramenta é útil para o desempenho das suas atividades, o profissional irá tirar proveito dessa propriedade, agregando valor as suas atividades profissionais (DAVIS et al., 1989).

\subsection{Percepção dos professores-tutores quanto a Importância Direcionada a Ação}

O constructo Importância Direcionada a Ação, consiste na junção das categorias "Atitude no Sentido de Usar" e "Intenção Comportamental para Usar", conforme já citado na parte metodológica. Esse constructo avaliou o sentimento e a intensão que o indivíduo demonstra ter para desempenhar determinado comportamento como prioridade em seu trabalho. Para análise dessa categoria foram elaboradas as afirmativas listadas na Tabela 2, nas quais os professores-tutores tiveram que manifestar concordância ou discordância.

As respostas dos professores-tutores demonstram que o SIGAA é uma ferramenta importante para a funcionalidade da $\mathrm{EaD}$, tendo em vista que a maioria o caracterizou como um sistema inovador, com ferramentas eficientes e eficazes.

Além disso, percebe-se que os respondentes se sentem motivados a explorar o sistema, e a utilizá-lo como prioridade, mesmo diante de outras ferramentas de comunicação. Os resultados da pesquisa vai de encontro as colocações de Davis et al. (1989) ao afirmar que a ação dos indivíduos não é relevante em ambientes em que o uso da tecnologia é obrigatório como no caso do SIGAA, pois percebe-se grande relevância no sentido da busca por tornar-se um usuário expertise, mesmo não sendo necessário tantos conhecimentos para operacionalizar o sistema no cotidiano do professor-tutor. 
Tabela 2 Importância Direcionada a Ação.

\begin{tabular}{|c|c|c|c|c|c|}
\hline Variáveis & $\begin{array}{l}\text { Discordo } \\
\text { Totalmente }\end{array}$ & $\begin{array}{c}\text { Discordo } \\
\text { Parcialmente }\end{array}$ & Indiferente & $\begin{array}{c}\text { Concordo } \\
\text { Parcialmente }\end{array}$ & $\begin{array}{l}\text { Concordo } \\
\text { totalmente }\end{array}$ \\
\hline $\begin{array}{l}\text { Realizar minhas atividades através das } \\
\text { ferramentas do SIGAA é uma ideia } \\
\text { inovadora. }\end{array}$ & $2,41 \%$ & $1,20 \%$ & $9,64 \%$ & $34,94 \%$ & $51,81 \%$ \\
\hline $\begin{array}{l}\text { Considero o SIGAA e suas ferramentas um } \\
\text { sistema de informação positivo, eficiente e } \\
\text { eficaz. }\end{array}$ & $1,20 \%$ & $6,02 \%$ & $2,41 \%$ & $45,78 \%$ & $44,58 \%$ \\
\hline $\begin{array}{l}\text { O SIGAA é compatível com outros } \\
\text { sistemas de informação que uso no meu } \\
\text { cotidiano. }\end{array}$ & $6,02 \%$ & $10,84 \%$ & $9,64 \%$ & $36,14 \%$ & $37,35 \%$ \\
\hline $\begin{array}{l}\text { Pretendo explorar ao máximo as } \\
\text { ferramentas do SIGAA e tornar-me um } \\
\text { usuário expertise. }\end{array}$ & $0,00 \%$ & $2,41 \%$ & $7,23 \%$ & $28,92 \%$ & $61,45 \%$ \\
\hline $\begin{array}{l}\text { Mesmo quando houver outras opções para a } \\
\text { realização das minhas atividades, o SIGAA } \\
\text { será sempre minha prioridade. }\end{array}$ & $2,41 \%$ & $4,82 \%$ & $8,43 \%$ & $38,55 \%$ & $45,78 \%$ \\
\hline
\end{tabular}

Fonte: dados da pesquisa, jun. 2016.

A intenção comportamental de usar uma ferramenta tecnológica é influenciada pela facilidade de manuseio da tecnologia (VENKATESH, 2000). Portanto, o comportamento dos professores-tutores em relação ao uso do SIGAA demonstra que o sistema é útil e de fácil uso, além de agregar funcionalidades capazes de englobar diversos aspectos do trabalho do professor-tutor, o que incentiva esses profissionais a utilizá-lo em sua jornada.

\subsection{Percepção dos professores-tutores quanto a Disposição Comportamental para Uso Real do Sistema}

Os professores-tutores que atuam na modalidade a distância se revelaram quanto a Disposição Comportamental para Uso Real do Sistema, em relação ao SIGAA, ao acederem ou discordarem das afirmativas listadas na Tabela 3:

Tabela 3 Disposição comportamental para uso real do sistema.

\begin{tabular}{|c|c|c|c|c|c|}
\hline Variáveis & $\begin{array}{c}\text { Discordo } \\
\text { Totalmente } \\
\end{array}$ & $\begin{array}{c}\text { Discordo } \\
\text { Parcialmente } \\
\end{array}$ & Indiferente & $\begin{array}{c}\text { Concordo } \\
\text { Parcialmente }\end{array}$ & $\begin{array}{r}\text { Concordo } \\
\text { totalmente }\end{array}$ \\
\hline $\begin{array}{l}\text { Procuro resolver as pendências via } \\
\text { SIGAA no dia em que elas surgem. }\end{array}$ & $2,41 \%$ & $7,23 \%$ & $3,61 \%$ & $38,55 \%$ & $48,19 \%$ \\
\hline $\begin{array}{l}\text { Atualizo as minhas atividades no } \\
\text { SIGAA diariamente. }\end{array}$ & $2,41 \%$ & $3,61 \%$ & $3,61 \%$ & $51,81 \%$ & $38,55 \%$ \\
\hline $\begin{array}{l}\text { Cumpro a minha carga horária todos os } \\
\text { dias online no SIGAA. }\end{array}$ & $1,20 \%$ & $6,02 \%$ & $3,61 \%$ & $46,99 \%$ & $42,17 \%$ \\
\hline
\end{tabular}

Fonte: dados da pesquisa, jun. 2016. 
Diante dos resultados, pode-se afirmar que o sistema é efetivamente utilizado pelos profissionais em sua jornada laboral. Nesse cenário positivo, o uso da tecnologia se solidifica corroborando para que haja praticidade no trabalho dos professores-tutores, viabilizando auxílio aos alunos nos processos do ensino-aprendizagem, além de proporcionar benefícios para a gestão educacional no âmbito da EaD.

A maioria das organizações são dependentes das tecnologias de comunicação e informação. A existência delas estão condicionadas a aplicação efetiva desses sistemas (NAKAMURA, 2016), por esse motivo, a avaliação do indicador aqui referenciado é fundamental para a continuidade da $\mathrm{EaD}$.

\subsection{Percepção dos coordenadores quanto à aceitação e utilização do SIGAA}

Para um maior grau de certeza da pesquisa foram realizadas entrevistas com os cinco coordenadores dos cursos que possuem mais professores-tutores na modalidade à distância, conforme Tabela 01 .

Os coordenadores entrevistados foram os de: administração, filosofia, letras-português, letras-inglês e sistema de informação. Estes cursos detêm 55,88\% do total de professorestutores do CEAD e 59,04\% do total de profissionais da amostra analisada. As entrevistas foram feitas a fim de saber se os professores-tutores realmente demonstram, na percepção dos gestores, aceitação às tecnologias em suas ações cotidianas enquanto profissionais da IES. O resultado da pesquisa quantitativa foi confirmado pelos coordenadores, que afirmaram por unanimidade que os seus respectivos colaboradores são profissionais que manuseiam bem as tecnologias, em especial o SIGAA.

Os coordenadores afirmaram ainda oferecer capacitações periódicas, para sanar as dificuldades com o sistema e acompanhamento de supervisão ao trabalho, para que este seja feito dentro dos prazos e da metodologia adotada pelos respectivos cursos. Nesse contexto, pode-se observar que os professores-tutores do CEAD não apresentam rejeição ao SIGAA, ratificando o elevado grau de aceitação e utilização do sistema na percepção desses profissionais e dos gestores.

Deve-se ressaltar, por conseguinte, que o CEAD iniciou suas atividades em 2006 e, na época, o sistema utilizado era a Plataforma Moodle, porém em 2014 houve a transição para o SIGAA, o que trouxe uma nova experiência tecnológica para os profissionais da EaD. $\mathrm{Na}$ visão dos coordenadores, os tutores sentiram a mudança, tendo em vista que o Moodle é uma 
plataforma mais "amigável”, por ser flexível quanto ao design e às possibilidades de alteração pela criatividade dos usuários, enquanto que o SIGAA é uma plataforma menos flexível nesse aspecto, por apresentar um design padronizado, não permitindo mudanças pelos usuários, mas, se alguma sugestão de inovação for proposta pelos usuários, somente será implementada pelos administradores do sistema, no caso o Núcleo de Tecnologia da Informação (NTI).

Além disso, há ferramentas no SIGAA que poderiam contribuir com o trabalho, mas não são utilizadas por conta das limitações de infraestrutura nos pólos. Isso ratifica o pensamento de que nem todos os docentes sentem-se motivados em utilizar as ferramentas tecnológicas no formato em que têm sido demandas pelas organizações (CHEN, 2004).

\section{CONSIDERAÇÕES FINAIS}

A introdução de tecnologias no ambiente de trabalho na esfera de uma organização pública é um grande desafio, tendo em vista as raízes culturais sólidas existentes nessas instituições. Nesse contexto, o trabalho docente nas universidades públicas vem sofrendo muitas mudanças com a massificação dos cursos à distância, caracterizados pela inserção de tecnologias no ambiente laboral do docente.

A pesquisa investigou, a partir dos construtos do Modelo de Aceitação de Tecnologia (TAM), a percepção dos professores-tutores dos cursos a distância do Centro de Educação Aberta e à Distância (CEAD) da Universidade Federal do Piauí (UFPI) quanto ao grau de aceitação e utilização efetiva das ferramentas tecnológicas do Sistema Integrado de Gestão de Atividades Acadêmicas (SIGAA).

Como contribuição do estudo, constatou-se que as variáveis externas como: o tempo de experiência dos docentes com o uso da tecnologia aplicada ao ensino; o ensinar na modalidade a distância; o pouco tempo de trabalho na Instituição de Ensino Superior (IES) e a falta de treinamento, para alguns profissionais, tornam possível se entender questões sobre a percepção quanto à utilização do Sistema.

Contudo, em relação aos construtos comportamentais de utilização de Sistemas de Informação (SI), verificou-se pelos indicadores propostos que os professores-tutores demonstram significativa aceitação e interesse em utilizar o sistema em estudo.

Os resultados obtidos com a pesquisa demonstram, portanto, que a percepção dos professores-tutores que atuam na modalidade a distância do CEAD validam os construtos propostos por Davis (1989), pois são capazes de prever a aceitação do SIGAA e porque os 
indivíduos tendem a utilizar ou não determinada tecnologia de acordo com a possibilidade de melhorar seu desempenho profissional com o menor esforço despendido.

De certo, o domínio do SIGAA e a familiarização com as diversas ferramentas tecnológicas que contribuem para a fluidez no processo de ensino-aprendizagem são de extrema importância para o trabalho nas IES que utilizam a modalidade EaD. Assim, para trabalhos futuros, sugere-se analisar fatores externos como a evolução percebida nos cursos pesquisados, a avaliação de desempenho docente, o feedback dos discentes e as avaliações internas e externas dos cursos e da instituição, consoantes os eixos e as dimensões do Sistema Nacional de Avaliação da Educação Superior (SINAES).

Considerando-se a natureza exploratória da pesquisa, incorporada às suas limitações, não seria criterioso sugerir prescrições. Todavia, a pesquisa pode servir como um limiar para novos estudos para os gestores do CEAD/UFPI verificarem os fatores que podem ser melhorados nas políticas de treinamento e capacitação dos profissionais e na busca do aprimoramento dos Sistemas Integrados de Gestão (SIG), entre eles o SIGAA, para que este possa disponibilizar ferramentas mais atrativas do ponto de vista da interação alunos/tutores/gestores, melhorando o comprometimento com o processo ensinoaprendizagem e diminuindo as dificuldades existentes na aceitação e no uso efetivo da tecnologia na gestão educacional em todas as suas vertentes.

\section{REFERÊNCIAS}

ALMEIDA, M. E. B. de. Tecnologia e educação a distância: abordagens e contribuições dos ambientes digitais e interativos de aprendizagem. 2012. In: Revista Brasileira de Educação a Distância. Rio de Janeiro, n. 110, ano 20, 2012.

BRADLEY, J. The technology acceptance model and other user acceptance theories. In: Handbook of research on contemporary theoretical models in information systems. IGI Global Disseminator of Knownledge, 2009, cap. XV, p. 277-294. Disponível em: $<$ http://www.igi-global.com/chapter/technology-acceptance-model-other-user/35835>. Acesso em: jun. 2016.

BUENO, U.; ZWICKER, R.; OLIVEIRA, M. A. de. Um estudo comparativo do modelo de aceitação de tecnologia aplicado em sistemas de informações e comércio eletrônico. In: I

Congresso Internacional de Gestão de Tecnologia e Sistemas de Informação - I CONTECSI. 2004. São Paulo, SP, 21-23 de junho de 2004. São Paulo: USP, 2004.

CARVALHO, M. A. de. Aceitação e intenção de uso do mobile learning: modelagem e teste empírico com alunos de ensino superior. Dissertação (Mestrado) - Pontifícia Universidade Católica do Rio de Janeiro, Departamento de Administração, 2013. 
CARVALHO, M. L. A. de; FREITAS, A. S. de; RAMOS, A. S. M.; NASCIMENTO, T. C.; FERREIRA, J. B. Fatores que afetam a intenção em continuar o uso do e-learning: um estudo com professores de uma universidade federal. In: Revista Administração em Diálogo, vol. 15, no 1, Jan/Fev/Mar/Abr/2013, p. 139-164. ISSN 2178-0080. Programa de Estudos PósGraduados em Administração : PUC-SP, 2013.

CASTELLS, M. A sociedade em rede. $3^{\text {a }}$. ed. São Paulo : Paz e Terra, 2000, v. 1.

CHEN, L.-L. Pedagogical strategies to increase pre-service teachers' confidence in computer learning. Educational Technology \& Society, 7, 2004.

DAVIS, F. D. Perceived usufulness, perceived ease of use, and user acceptance of information technology. MIS Quarterly, v. 13, n. 3, p. 319-339, 1989.

DAVIS, F. D.; BAGOZZI, R.; WARSHAW, P. R. User acceptance of computer technology: a comparison of two theoretical models. Management Science, v. 5, n. 8, p. 982-1003, 1989.

HUERTAS, A. Teaching and learning logic in a virtual learning environment. Logic Journal of IGPL, Oxford, v. 15, n. 4, p. 321-331, 2007.

HAIR JR, et al. Análise multivariada de dados. 5 ed. Porto Alegre: Bookman, 2005.

LIMONGI-FRANÇA, A. C. Qualidade de vida no trabalho - QVT: conceitos e práticas nas empresas da sociedade pós-industrial. ${ }^{\mathrm{a}}$. ed. $4^{\mathrm{a}}$. reimp. São Paulo : Atlas, 2009.

MACHADO, P. de A.; BELLINI, C. G. P.; LEITE, J. C. de L. Adoção e uso de inovação tecnológica em educação a distância: estudo sobre integração de TAM e IDT. 2011. In:

XXXV Encontro da ANPAD. Rio de Janeiro: EnANPAD, 4 a 7 set. 2011.

NAKAMURA, Adi, The Evolving Role of Information Systems and Technology in Organizations: A Strategic Perspective. Peppard-c01.indd, 2016. Disponível em: $<$ https://www.esmt.org/sites/default/files/peppard-c01.pdf $>$ Acesso em maio de 2018.

OLIVEIRA, M. F. de. Metodologia científica: um manual para a realização de pesquisas em administração. Catalão : UFG, 2011. 72p.: Il.

PARK, S. Y. An Analysis of the Technology Acceptance Model in Understanding University Students' Behavioral Intention to Use e-Learning. 2009. In: Educational Technology \& Society, 12 (3), 150-162, 2009.

SILVA, P. M. da; DIAS, G. A.; ALMEIDA, J. R. de. Modelo de Aceitação de Tecnologia (TAM) aplicado ao Sistema de Informação da Biblioteca Virtual em Saúde (BVS) nas escolas de medicina da região metropolitana do Recife. Recife. 2013. Disponível em: $<$ http://enancib.ibict.br/index.php/enancib/xenancib/paper/viewFile/3371/2497>. Acesso em: 07 dez. 2015. 
SILVA, A. A. C. e. Um estudo da Aplicação do Modelo de Aceitação de Tecnologias na Educação Superior com Foco nos Ambientes Virtuais de Aprendizagem. 2014. In: Revista Científica em Educação a Distância. EAD em foco. Disponível em: <www.eademfoco.com.br>. v. 4, nº 2, 2014. Acesso em: mai. 2016.

SILVA, P.; PIMENTEL, V.; SOARES, J. A utilização do computador na educação: aplicando o Technology Acceptance Model (TAM). 2012. In: Biblionline. João Pessoa, v. 8, n. esp. p. 263-272, 2012.

TENÓRIO, F. G. Tecnologia da informação transformando as organizações e o trabalho. Rio de Janeiro : Editora FGV, 2007. 216p.

TEO, T. Technology Acceptance Research in Education. In: TEO, T. (org.) Technology Acceptance in Education. Roterdã : Sense Publishers, 2011.

TEZZA, M. M. Aceitação e resistência à educação a distância: um estudo em uma empresa do setor metalúrgico. 2012. In: XXXVI Encontro da ANPAD. Rio de Janeiro : EnANPAD, 22 a 26 set. 2012.

UFRN. Sistemas Institucionais Integrados de Gestão (SIG). Superintendência de Informática da Universidade Federal do Rio Grande do Norte (SINFO/UFRN). s/d. Disponível em: $<$ https://www.info.ufrn.br/wikisistemas/doku.php?id=suporte $>$. Acesso em: maio. 2016.

VENKATESH, V. Determinants of Perceived Ease of Use: Integrating Perceived Behavioral Control, Computer Anxiety and Enjoyment into the Technology Acceptance Model.

Information Systems Research, v. 11, n. 4, p. 342-365, 2000.

VENKATESH, V.; MORRIS, M. G.; DAVIS, G. B.; DAVIS, F. D. User acceptance of information technology: toward a unified view. MIS Quarterly, v. 27, n. 3, p. 425-478, 2003.

ZANELLA, L. C. H. Metodologia de estudo e de pesquisa em administração. Florianópolis : Departamento de Ciências da Administração. UFSC: Brasília, CAPES, UAB. 2009. 164p.: Il. 